\title{
Lot Sizing and Scheduling in Flow Shop with Sequence- Dependent Setups and Backlogging
}

\author{
M.Babaei \\ Department of Industrial \\ Engineering, Faculty of \\ Engineering, TarbiatMoallem \\ University, Karaj, Iran
}

\author{
M.Mohammadi \\ Department of Industrial \\ Engineering, Faculty of \\ Engineering, TarbiatMoallem \\ University, Karaj, Iran
}

\author{
S.M.T.FatemiGhomi \\ Department of Industrial \\ Engineering, Amirkabir \\ University of Technology, \\ Tehran, Iran
}

\begin{abstract}
Lot sizing and scheduling in flow shop has been considered in this paper. Our study includes a multi-level and multi-period capacitated lot sizing and scheduling problem (CLSP) with sequence-dependent setups, setups carry over in flow shop. In manufacturing environments backlogging is unavoidable. If the production capacity is infinite, any demand can be satisfied on time. But in the real world, production capacity is not infinite so some demand may not be satisfied on time. In this condition backlogging arises naturally. CLSP problems with considering backlogging has been studied fewer by researchers. Also, the sequence-dependent setups and setups carry over are two important events that occur occasionally in factories. In this study an exact mixed integer problem (MIP) in a flow shop CLSP problem with considering sequence-dependent setups, setups carry over and backlogging has been formulated. Our formulation is presented to optimize objective function (including, inventory costs, product costs and setup cost). Since the CLSP problems are extremely NP-hard a lower bound is developed and compared against the optimal solution.
\end{abstract}

\section{General Terms}

Lot sizing and Scheduling.

\section{Keywords}

Flow shop, sequence-dependent setups, backlogging, lot sizing and scheduling.

\section{INTRODUCTION}

Scheduling and lot sizing is applicable in diverse area, especially in manufacturing sector. Smooth and cost-efficient running of a factory often depends on its manager's ability to select appropriate lot sizes and production schedules[1].

Since the hypothesis of improving performance through investments in new equipment and tools for quicker set-up times was rejected by the company, the solution was unavoidably oriented towards developing a very effective production planning model that also guarantees computational times consistent with rapid re-planning [2]. After proposing Economic Order Quantity (EOQ), Lot sizing problems have been studied widely. Since then other works have been developed in order to relax the restrictive assumption of the EOQ model. the Economic Lot Scheduling Problem (ELSP) takes into account capacity constraints.

More recent works have studied combination of lot sizing and scheduling simultaneously. In the literatures has been seen so many article about scheduling and lot sizing. Models of lot sizing and scheduling are divided in the literature into small bucket and big bucket problems[3].

Small bucket problems break the planning horizon in small time periods which limits the number of products manufactured in a single period. Small bucket problems are divided in the literature into discrete lot sizing and scheduling problem (DLSP) and proportional lot sizing and scheduling problem (PLSP). In DLSP at most one product can be manufactured in a single period, but PLSP allows up to two products [4].

Also, one of the most difficult lots sizing problem is the capacitated lot-sizing and scheduling problem (CLSP) with sequence-dependent setup costs and non-zero setup times. The CLSP is encountered when one or more machines (or production facilities) are used to meet forecasted demand for multiple products over multiple periods. Factory managers must decide which products to make in which periods, and the exact production sequence and production quantities, in order to minimize the sum of setup and inventory holding costs. What makes this problem particularly difficult to solve in many applications is the fact that capacity is tight, setup costs are large and sequence-dependent, and setup times are non-zero [5].

Florian et al. [5] have proved that the single-item CLSP is NPhard. Later, Bitran and Yanasse[6] showed that even special cases which are solvable in polynomial time become $N P$-hard through the introduction of a second item. Trigeiro et al. [7] pointed out that for the CLSP with setup times the question whether a feasible schedule exists is already NP-complete. Trigeiro et al. [7] referred to bin packing as a special case of the CLSP with setup times. For the proof of $N P$-completeness see Garey and Johnson [8]. As the MLCLSP and the MLCLSPL can be reduced to the CLSP with setup times by setting some parameters to 0 , they are at least as hard to solve and hence also $N P$-hard.

Traditional CLSP models did not consider the backlogging. Those suppose that each demand must be delivered on time at the end of each period. In this study this restrictive constraint to be relaxed. In the real world we cannot be sure that every demand of a product could be produce on time because of unpredictability of manufacturing environments. For example, if the production rate of the machine is infinite, any demand can be satisfied on time. But if the production rate of the machine is finite, some demand may not be satisfied on time [9], and then backlogging would be occurred.

Perishable goods are an important part of inventories and perishability and backlogging can be considered to be 
complementary phenomena. When a good is highly perishable, the demand may need to be backlogged to contain costs due to deterioration. This way, the losses due to deterioration can be avoided and the good can be procured in an economic manner [10].

While only few lot-sizing approaches consider the possibility of back-ordering, it is of great importance in practical settings: If capacity is limited, some products may have to be back-ordered. However, it is no satisfactory result to understand that some demand volume cannot be produced on time. In fact, the question is, which demand volumes should be back-ordered and which should not? [11].

Despite its importance in practical settings, only few researchers have addressed capacitated lot-sizing problems with backordering. Examples include the articles byDaniel Quadt, Heinrich Kuhn [11],Pochet and Wolsey [12], Millar and Yang [13], Karimi et al. [15], and. In some cases demand cannot be backlogged, but will be totally or partially lost. This situation is considered by Absi and Kedad-Sidhoum[16].

Further complication is the fact that a product can be set up at the end of one time period and the actual production start in the next time period. We call this property setup carry over[17]. In a manufacturing environment, there are instances where developing a feasible schedule is only possible when setup states are carried over from one period to another. Setup carry over is the continuation of a production run from one period to the next with- out an additional setup. Setup carry over in big-bucket models is concerned with "partial sequencing" of items. The sequence of items scheduled between the first and last does not affect the total required setup time unless the items are sequence dependent, where setups can be done in any order. The complexity of modeling setup carry over in CLSP problems is why it has not received much attention in the literature [18].

Diwakar Gupta and Thorkell Magnusson[1], have presented the single machine capacitated lot-sizing and scheduling problem (CLSP) with sequence-dependent setup costs and non-zero setup times, with the additional feature that setups may be carried over from one period to the next, and that setups are preserved over idle periods. They provided an exact formulation of this problem as a mixed-integer program.

Florian Sahlinga, , LisbethBuschkühlb, Horst Tempelmeierb and Stefan Helber[19] presented a new algorithm for the dynamic multi-level capacitated lot sizing problem with setup carry-overs (MLCLSP-L). The MLCLSP-L is a big-bucket model that allows the production of any number of products within a period, but it incorporates partial sequencing of the production orders in the sense that the first and the last products produced in a period are determined by the model.

Ik-Soo Shim, Hyeok-Chol Kim, Hyoung-Ho Doha and Dong-Ho Lee [20]considerd a single machine capacitated lot-sizing and scheduling problem. They considered sequence-dependent setup costs that depend on the type of the lot just completed and on the lot to be processed. The setup state preservation, i.e., the setup state at the end of a period is carried over to the next period, is also considered.

In practice various methods can be used to solve the problem of simultaneous lot sizing and scheduling. These differ with regard to their specific features. Among the characteristic features of the models for lot sizing and scheduling are the segmentation of the planning horizon, the time dependence of the model parameters, the information degree of the model parameters, the number of products and production stages, the production structure and the capacity restrictions.

Because of NP-hardness these models, often lower bounds, heuristics and metaheuristics are proposed. Diwakar Gupta and Thorkell Magnussonhave developed a heuristic for solving large problem instances. This is coupled with a procedure for obtaining a lower bound on the optimal solution. They have carried out a computational study to test the accuracy of several different lower bounding linear relaxations and the approximate solution obtained by the heuristic. . In this paper we present an exact formulation with a lower bound. We relax constraints to develop a lower bound.

The rest of the paper is organized as follows. Section "mathematical model" includes a definition of the problem and assumptions; an illustrative example is presented too. In the section "Developing of lower bound", one lower bound is developed. Section "Numerical examples" reports the numerical examples for lower bound and compares its resultsagainst obtained results of the exact model. In the last section "Conclusions" is devoted to the concluding remarks and recommendations for future studies.

\section{MATHEMATICAL MODEL}

In this section we describe model and assumptions. As mentioned above, one of the most difficult lots sizing problem is the capacitated lot sizing and scheduling problem (CLSP) with sequence-dependent setup costs and non-zero setup times and these problems are practical in so many sector. For example, glass container and some chemical industries [17].

Only few researchers have addressed capacitated lot sizing problems with back-ordering Daniel Quadt, Heinrich Kuhn [11], also the complexity of the modeling setup carry over in CLSP problems result in the not much attention in the literatures [18]. So in this paper we consider a lot sizing and scheduling in flow shop with sequence-dependent setups, setups carry over and backlogging.

Assumptions of model follow.

- Several products are produced in a flow shop structure

- Each machine can produce only one product at the same time.

- Capacity of each machine is constrained.

- Setups are sequence-dependent and when a setup occurs, setup cost and setup time is charged.

- A setup of a machine must be complete in a period

- $\quad$ There must be precisely $N$ (number of products) setups in each period on each machine, even if a setup is just from a product to itself. Setups follow the so-called triangular inequalities, i.e. it is never faster to change over from one product to another by means of a third product: otherwise over $N$ setups in a time period could be obtained. Since a setup time (and cost) from a product to itself is zero, note that the model does not force a machine to have exactly $N$ positive-time (and cost) setups but rather up to $N$ such setups. The remaining zero-time (cost) setups are modeling phantoms and do not exist in reality (Clark and Clark 
2000, Clark 2003). This feature makes possible for a lot size, or production run, to continue over consecutive time periods without incurring real setup for later period (setup carry over).

- The required resources and parts must be ready for production.

- External demand exists for final product but it is possible that demand is not satisfied at the end of each period, and then backlogging could occur.

- Backlogging is permitted. If backlogging occurs at a period it could be responded at other period (back order).

- There is no lead time between the different production levels.

- A component cannot be produced earlier in a period than the production of its required component is finished, this assumption remains true even if there is inventory of the required component. In other words, production on a production level can only start if all products from the previous production level are available; this is called vertical interaction

- To guarantee the vertical interaction, idleness between each setup and its production is defined with the help of shadow product (Fandel and Stammen- Hegene 2006).

- $\quad$ There are no demand and no storage costs for shadow products.

- At the beginning of the planning horizon each machine is setup for a defined product.

For formulating our model we use indices and parameters that follow.

Indices

$i, j, \quad$ Production type.

$n, n^{\prime}, n^{\prime \prime}$ Designation for a specific setup number.

$m \quad$ Level of production.

$t \quad$ Period.

Parameters

T Planning horizon.

$N \quad$ Number of different products.

$M \quad$ Number of production levels/number of machines.

bigM A large real number.

$C_{m, t} \quad$ Available capacity of machine $m$ in period $t$ (in time units).

$d_{j, t} \quad$ External demand for product $j$ at the end of period $t$ (in units of quantity). $h_{j, m}^{+} \quad$ Storage costs unit rate for product $j$ in level $m$.

$h_{j, t}^{-} \quad$ Shortage costs unit rate for product $j$ at the end of period $t$.

$b_{j, m} \quad$ Capacity of machine $m$ required to produce a unit of product (or shadow product) $j$ (in time units per quantity units).

$P_{j, m, t} \quad$ Production costs to produce one unit of product $j$ on machine $m$ in period $t$ (in money unit per quantity unit).

$S_{i, j, m} \quad$ Sequence-dependent setup time for the setup of the machine $m$ from production of product $i$ to production of product $j$ (in time units); for $i \neq j, S_{i, j, m} \geq 0$ and $i=$ $j, S_{i . j . m}=0$.

$W_{i, j, m} \quad$ Sequence-dependent setup cost for the setup of the machine $m$ from production of product $i$ to production of product $j$ (in money units); for $i \neq j, W_{i, j, m} \geq 0$ and $i=j, W_{i . j . m}=0$.

$j_{0 m} \quad$ The starting setup configuration on machine $m$.

\section{Decision variables}

$I_{j, m, t}^{+} \quad$ Stock of product $j$ at level $m$ at the end of period $t$.

$I_{j, t}^{-} \quad$ Shortage of product $j$ at the end of period $t$.

$y_{i, j, m, t}^{n} \quad$ Binary variable, which indicates whether the $n$th setup on machine $m$ in period $t$ is from product $i$ to product $j\left(y_{i, j, m, t}^{n}=1\right)$ or not $\left(y_{i, j, m, t}^{n}=0\right)$.

$x_{i, j, m}^{n} \quad$ Quantity of product $j$ produced after $n$th setup on machine $m$ in period $t$.

$q_{i, j, m}^{n} \quad$ Shadow product: the gap (in quantity units) between $n$th setup (to product $j$ ) on machine $m$ in period $t$ and its related production in order to ensure that direct predecessor of this product (production of product $j$ on machine $m$ in period $t$ ) has been completed. In other words, idle time (in quantity units) before production of product $j$ on machine $m$ in period $t$ in order to guarantee vertical interaction.

\section{1 formulation}

$\min \sum_{n=1}^{N} \sum_{j=1}^{N} \sum_{i=1}^{N} \sum_{m=1}^{M} \sum_{t=1}^{T} W_{i, j, m} \cdot y_{i . j, m, t}^{n}+\sum_{n=1}^{N} \sum_{j=1}^{N} \sum_{m=1}^{M} \sum_{t=1}^{T} P_{j, m, t} \cdot x_{j, m, t}^{n}+\sum_{j=1}^{N} \sum_{m=1}^{M} \sum_{t=1}^{T} h_{j, m}^{+} \cdot I_{j, m, t}^{+}+\sum_{j=1}^{N} \sum_{t=1}^{T} h_{j, t}^{-} \cdot I_{j, t}^{-}$ 
Subject to

$$
\begin{aligned}
& d_{j, t}=I_{j, M, t-1}^{+}+\sum_{n=1}^{N} x_{j, M, t}^{n}-I_{j, M, t}^{+}-I_{j, t-1}^{-}+I_{j, t}^{-}, \quad j=1, \ldots, N, \quad t=1, \ldots, T \\
& I_{j, m, t-1}^{+}+\sum_{n=1}^{N} x_{j, m, t}^{n}=I_{j, m, t}^{+}+\sum_{n=1}^{N} x_{j, m+1, t}^{n}, \quad j=1, \ldots, N, \quad m=1, \ldots, M-1, \quad t=1, \ldots, T \\
& \operatorname{bigM} .\left(\sum_{i=1, i \neq j\left(f \circ \operatorname{sor}_{\left(n^{\prime}>1\right)}\right.} y_{i, j, m, t}^{n^{\prime}}-1\right)+\sum_{n=1}^{n^{\prime}} \sum_{i=1}^{N} \sum_{k=1}^{N} y_{i, j, m, t}^{n} \cdot S_{i, k, m}+\sum_{n=1}^{n^{\prime}} \sum_{k=1}^{N} b_{k, m} \cdot q_{k, m, t}^{n}+\sum_{n=1}^{n^{\prime}} \sum_{k=1}^{N} b_{k, m} \cdot x_{k, m, t}^{n} \\
& \leq \operatorname{big} M \cdot\left(1-\sum_{i=1, i \neq j\left(f o r\left(n^{\prime \prime}>1\right)\right.}^{N} y_{i, j, m+1, t}^{n^{\prime \prime}}\right)+\sum_{n=1}^{n^{\prime \prime}} \sum_{i=1}^{N} \sum_{k=1}^{N} y_{i, j, m+1, t}^{n} \cdot S_{i, k, m+1}+\sum_{n=1}^{n^{\prime \prime}} \sum_{k=1}^{N} b_{k, m+1} \cdot q_{k, m+1, t}^{n} \\
& +\sum_{n=1}^{n^{\prime \prime}-1} \sum_{k=1}^{N} b_{k, m+1} \cdot x_{k, m+1, t}^{n}, \quad j=1, \ldots, N \text {, } \\
& n^{\prime}=1, \ldots, N, n^{\prime \prime}=1, \ldots, N, \quad m=1, \ldots, M-1, \quad t=1, \ldots, T
\end{aligned}
$$

$$
\begin{aligned}
& \sum_{n=1}^{n} \sum_{i=1}^{N} \sum_{j=1}^{N} y_{i, j, m, t}^{n} \cdot S_{i, j, m}+\sum_{n=1}^{n} \sum_{j=1}^{N} b_{j, m} \cdot x_{j, m, t}^{n}+\sum_{n=1}^{n} \sum_{j=1}^{N} b_{j, m} \cdot q_{j, m, t}^{n} \leq C_{m, t} \quad m=1, \ldots, M, \quad t=1, \ldots, T \\
& x_{j, m, t}^{n} \leq\left(\frac{C_{m, t}}{b_{m, t}}\right) . \sum_{\substack{i=1, i \neq j(f o r(n>1) \\
t=1, \ldots, T}}^{N} y_{i, j, m, t}^{n}, n=1, \ldots, N, \quad j=1, \ldots, N, \quad m=1, \ldots, M, \\
& q_{j, m, t}^{n} \leq\left(\frac{C_{m, t}}{b_{m, t}}\right) \cdot \sum_{i=1}^{N} y_{i, j, m, t}^{n}, \quad n=1, \ldots, N, \quad j=1, \ldots, N, \quad m=1, \ldots, M, \quad t=1, \ldots, T \\
& y_{j, i, m, 1}^{1}=0 j \neq j_{0 m}, \quad i=1, \ldots, N, \quad m=1, \ldots, M \\
& \sum_{i=1}^{N} y_{j_{0 m}, i, m, 1}^{1}=1, \quad m=1, \ldots, M \\
& \sum_{j=1}^{N} y_{j, i, m, t}^{n}=\sum_{k=1}^{N} y_{i, k, m, t}^{n+1} \quad i=1, \ldots, N, \quad n=1, \ldots, N, \quad m=1, \ldots, M, \quad t=1, \ldots, T
\end{aligned}
$$




$$
\begin{aligned}
& \sum_{j=1}^{N} y_{j, i, m, t-1}^{N}=\sum_{k=1}^{N} y_{i, k, m, t}^{1} i=1, \ldots, N, \quad n=1, \ldots, N, \quad m=1, \ldots, M, \quad t=2, \ldots, T \\
& y_{i, j, m, t}^{n}=0 \text { or } 1 \\
& I_{j, m, t}^{+}, I_{j, t}^{-}, x_{j, m, t}^{n}, q_{j, m, t}^{n} \geq 0 \\
& I_{j, m, 0}^{+}=0, \quad j=1, \ldots, N, \quad m=1, \ldots, M
\end{aligned}
$$

In our model, Equation (1), objective function, minimizes sum of the sequence-dependent setup costs, the storage costs, the production costs and the shortage cost. Equation (2) represents the shortage or storage at the end of each period. Constrain (3) ensures total of in-flows to each node is equal to of out-flows from that node.

Equation (4) guarantees within one period each typical product $j$ one machine $m$ is produced before its direct successor (product $j$ on machine $m+1$ ).

The left side of Equation (4) is equal to the time between the beginning of period $t$ and the end of production of product $j$ on machine $m$ if $n^{\prime}$ th setup in machine $m$ and period $t$ is from every product $i$ to product $j$ (for $n^{\prime}>1, i \neq j$ ), else it is a negative number. In other words, if $x_{j, m, t}^{n^{\prime}}$ cannot get a positive value the left side of Equation (4) would get a negative value. The right side of Equation (4) is equal to the time between the beginning of period $t$ and the beginning of production of product $j$ on machine $m+l$ if $n^{\prime \prime}$ th setup in machine $m+1$ and period $t$ is from every product $i$ to product $j$ (for $n^{\prime \prime}>1, i \neq j$ ), else it is a big number. In other words, if $x_{j, m+1, t}^{n^{\prime \prime}}$ cannot get a positive value, the right side of Equation (4) would get a big value.

The capacity constraints of machine during period are represented by equation (5). Equation (6) considers setups in

Table 1. Demand of product

production process. Equation (7) shows the relationship between shadow products and setups. Constraints (8) and (9) ensure that for each machine, the first setup at the beginning of the planning horizon is from a defined product. Equation(10) and (11) represent the relationship between successive setups. Equation (8) to (11) ensure that for each triple $(n, m, t)$ there is exactly one pair $(i, j)$ which $y_{i, j, m, t}^{n}=1$. The type of variables is defined by equations (12) and (13) and finally equation (14) indicates that at the end of planning horizon there is no on-hand inventory.

\subsection{Illustrative example}

A small problem has been presented to illustrate the model. We suppose a system with two serially-arranged machines that produce the products in flow shop environment. In addition, three products must be produced in two periods $(M=2, N=3$, $T=2)$. Product 1 is produced at the beginning of the planning horizon. Table 1.shows demand of the products. Unit production times and costs are shown in Table 2. Holding cost unit rates are shown in Table 3.Table 4.shows setup times and costs. Capacity of the machines is showed in Table 5. Table 6.shows backlogging cost unit rates.

With respect parameters LINGO software is utilized to find the optimal solution. Table 7.shows the optimal solution.

\begin{tabular}{cccc}
\hline & Product 1 & Product 2 & Product 3 \\
\hline Period 1 & 200 & 100 & 300 \\
\hline Period 2 & 200 & 300 & 100 \\
\hline
\end{tabular}

Table 2.Unit production times and costs.

\begin{tabular}{cccccccc}
\hline & \multicolumn{3}{c}{ Machine 1 } & \multicolumn{3}{c}{ Machine 2 } \\
\cline { 2 - 7 } & Product 1 & Product 2 & Product 3 & Product 1 & Product 2 & Product 3 \\
\hline Period 1 & 4.5 & 6 & 6 & 4.5 & 6 & 6 \\
\hline Period 2 & 4.5 & 6 & 6 & 4.5 & 6 & 6 \\
\hline
\end{tabular}

Table 3. Holding cost unit rate.

\begin{tabular}{cccc}
\hline & Product 1 & Product 2 & Product 3 \\
\hline Machine 1 & 3 & 2 & 4 \\
\hline Machine 2 & 3 & 2 & 4 \\
\hline
\end{tabular}


Table 4.Sequence-dependent setup times and costs.

\begin{tabular}{ccccccc}
\hline & \multicolumn{3}{c}{ Machine 1 } & \multicolumn{3}{c}{ Machine 2 } \\
\cline { 2 - 7 } & Product 1 & Product 2 & Product 3 & Product 1 & Product 2 & Product 3 \\
\hline Product 1 & 0 & 100 & 70 & 0 & 100 & 70 \\
\hline Product 2 & 90 & 0 & 80 & 90 & 0 & 80 \\
\hline Product 3 & 110 & 100 & 6 & 110 & 100 & 6 \\
\hline
\end{tabular}

Table 5.Capasity of machines.

\begin{tabular}{ccc}
\hline & Machine 1 & Machine 2 \\
\hline Period 1 & 4000 & 5000 \\
\hline Period 2 & 4000 & 5000 \\
\hline
\end{tabular}

Table 6. Backlogging cost unit rate.

\begin{tabular}{cccc}
\hline & Product 1 & Product 2 & Product 3 \\
\hline Period 1 & 10 & 18 & 20 \\
\hline Period 2 & 10 & 18 & 20 \\
\hline
\end{tabular}

Table 7. Non-Zero decision variables

$$
\begin{gathered}
y_{1,1,1,1}^{1}=1 \\
y_{1,3,2,1}^{2}=1 \\
y_{2,2,2,2}^{1}=1 \\
y_{3,2,1,1}^{3}=1 \\
x_{1,2,1}^{1}=200 \\
x_{2,2,1}^{3}=100 \\
x_{3,1,2}^{3}=122.5 \\
q_{1,2,1}^{1}=370 \\
I_{3,1}^{-}=22.5
\end{gathered}
$$

$$
\begin{gathered}
y_{1,1,2,1}^{1}=1 \\
y_{2,2,1,2}^{1}=1 \\
y_{2,2,2,2}^{2}=1 \\
y_{3,2,2,1}^{3}=1 \\
x_{2,1,1}^{3}=100 \\
x_{2,2,2}^{1}=300 \\
x_{3,2,1}^{2}=277.5 \\
q_{2,2,1}^{1}=397.5
\end{gathered}
$$

$$
\begin{gathered}
y_{1,1,2,1}^{1}=1 \\
y_{2,2,1,2}^{2}=1 \\
y_{2,3,1,2}^{3}=1 \\
x_{1,1,1}^{1}=200 \\
x_{2,1,2}^{1}=300 \\
x_{3,1,1}^{2}=277.5 \\
x_{3,2,2}^{3}=122.5 \\
I_{1,2}^{-}=200
\end{gathered}
$$

The optimal solution based on the parameters is showed in Table. 7. The sequence in both machines in Period 1 is 1-3-2 and in Period 2 is 2-3.The optimal objective function is 14350.

\section{DEVELOPING OF LOWER BOUND}

Florian et al. [5] have proved that the single-item CLSP is NPhard. So solving of these problems in medium or large scale would take a polynomial time. Then, for solving our formulation we need to develop lower bound which not obtains an optimum solution necessary but a good solution in reasonable time. In this section we present a lower bound by relaxing some of constraints. we relax binary variables as continuous variables between 0 to 1 . In this case, equation (4) does not impact on the problem because for non-integer values of $y_{i, j, m, t}^{n}$ the left side of Equation (4) would be negative and the right side of Equation (4) would be a big number, therefore with this relaxing, Equation (4) does not guarantee vertical interaction.

In this condition we do not have any binary variables. At the next section we present some numerical experiment to test and 
compare this lower bound against exact formulation. In all of examples, we consider 10 hours to reaching the solution (optimum solution or near optimum solution).

\section{NUMERICAL EXPERIMENTS}

In order to test the performance of lower bound, we establish several numerical examples. In these examples we compare solution of the exact model against lower bound according to computation tests and percentage of difference between exact and lower bound.

To solve the exact model and lower bound LINGO 8.0 is used. The models run in a personal computer with an Intel (R), Core (TM) 2 Duo CPU at $2.94 \mathrm{GHz}$.
The required parameters are provided from the following uniform distribution:

$b_{j, m} \approx U(5,10)$,

$d_{j, t} \approx U(500,1000)$,

$h_{j, t}^{+} \approx U(5,13)$,

$P_{j, m, t} \approx U(5,10)$,

$W_{j, m, t} \approx U(50,100)$,

$S_{j, m, t} \approx U(50,100)$,

$h_{j, t}^{-} \approx U(5,12)$,

$C_{m, t} \approx U(50000,100000)$,

The results of test are showed in Table. 8 .

Table 8.Comparison of optimal solution and lower bound.

\begin{tabular}{|c|c|c|c|c|c|}
\hline $\begin{array}{l}\text { Problem size } \\
\text { (N.M.T) }\end{array}$ & Optimal solution & $\begin{array}{c}\text { Computation times } \\
\text { (Seconds) }\end{array}$ & $\begin{array}{c}\text { Lower bound } \\
\text { solution }\end{array}$ & $\begin{array}{c}\text { Computation times } \\
\text { (Seconds) }\end{array}$ & Lower/optima \\
\hline 5.3 .3 & 171169 & 171090 & $99.95 \%$ & 66 & 1 \\
\hline 3.5 .3 & 115464 & 115464 & $100.00 \%$ & 1 & 0 \\
\hline 3.3 .5 & 2373522 & 2373552 & $99.99 \%$ & 322 & 1 \\
\hline
\end{tabular}

* Means that finding the optimum value requires more than 10 hours (36000 seconds).

- Means that feasible solution has not been found after 10 hours.

Table 8. Also shows these problems are extremely NP-hard. A problem with size 3.3 .5 only takes 322 seconds to finding optimum solution but the solution of a problem with size 5.5.5 has not been found even during 36,000 Seconds. The result shows in the medium scale, the lower bound obtain a good near optimum solution, even in an example with 3.5.3 optimum solution is reached by lower bound. This means this lower bound could be an alternative to solve these problems at least in the medium scales.

\section{CONCLUSION}

In this paper we consider both the backlogging and sequencedependent setups in flow shop environment. Sequencedependent setups have been studied not much, also the practical importance of backlogging the result in we study these issue together. We develop a MIP exact formulation. Because of NPhardness of the model we develop one lower bound. To test accuracy of proposed lower bound, some numerical examples have been developed. The solution obtained by lower bound against optimal solution showed accuracy of lower bound.

For future study we propose application heuristic and metaheuristic methods to solve this model. Considering to maintenance activities, constraint storage, machine breaking down and stochastic process time could be more contribution. The authors of this paper are studying about heuristic and metaheuristic methods, and developing a better lower bound.

\section{REFERENCES}

[1] Gupta and Magnusson 2005. The capacitated lot-sizing and scheduling problem with sequence-dependent setup costs and setuptimes. Computers \& Operations Research, 32(4), 727-747.

[2] FabrizioMarinelli, Maria Elena Nenni and Antonio Sforza. Capacitated lot sizing and scheduling with parallel machines and shared buffers: A case study in a packaging company.

[3] Eppen and Martin 1987. Solving multi-item capacitated lot-sizing problems using variable redefinition. Operations.

[4] Drexl and Kimms 1997. Lot sizing and scheduling, survey and extensions. European Journal of Operational Research, 99(2), 221-235.

[5] Florian M, Lenstra J, Kan AR (1980). Deterministic production planning: algorithms and complexity. Manage Sci 26:669-679.

[6] Bitran GR, Yanasse HH (1982). Computational complexity of the capacitated lot size problem.ManageSci 28:1174-1186.

[7] Trigeiro WW, Thomas LJ, McClain JO (1989). Capacitated lot sizing with setup times. Manage Sci 35:353-366.

[8] Garey M, Johnson D (1979). Computers and intractability: a guide to the theory of NP-completeness. Freeman, New York. 
[9] Yuyue Song a, Gin Hor Chan. Single item lot-sizing problems with backlogging on a single machine at a finite production rate.

[10] P.L. Abad. Optimal lot size for a perishable good under conditions of finite production and partial backordering and lost sale.

[11] Daniel Quadt, Heinrich Kuhn. Capacitated lot-Sizing and scheduling with parallel machines, back-orders, and setup carry-over.

[12] Yves Pochet and Laurence A. Wolsey. Lot size models with back-logging: Strong reformulations and cutting planes,Math Programming 40 (1988), 317-335.

[13] Harvey H. Millara and MinzhuYangb. Lagrangian heuristics for the capac- itated multi-item lot-sizing problem with backordering, Int J Prod Economics 34 (1994), 1-15.

[14] Ping-FengPaiE-mail. Solving the capacitated lot-sizing problem with backorder consideration, J Oper Res Soc 52 (2001), 952-959.

[15] Karimi,Ghomi, S M T Fatemil and Wilson J M. A tabu search heuristic for solving the CLSP with backlogging and set-up carry-over, J Oper Res Soc 57 (2006), 140147.

[16] Nabil Absi, SafiaKedad-Sidhoum. The multi-item capacitated lot-sizing problem with setup times and shortage costs, Eur J Oper Res 185 (2008), 1351-1374.
[17] Bernardo Almada-loboab; Diego Klabjanb; Maria Antóniacarravillaac; José F. Oliveiraac. Single machine multi-product capacitated lot sizing with sequencedependent setups.

[18] C. Oztürk, A.M. Ornek. Capacitated lot sizing with linked lots for general product structures in job shops.

[19] Florian Sahlinga, LisbethBuschkühl, Horst Tempelmeierb and Stefan Helber. Solving a multi-level capacitated lot sizing problem with multi-period setup carry-over via a fix-and-optimize heuristic

[20] Ik-Soo Shim, Hyeok-Chol Kim, Hyoung-Ho Doh and Dong-Ho Lee. A two-stage heuristic for single machine capacitated lot-sizing and scheduling with sequencedependent setup costs.

[21] Merece, C. Fonton, G, 2003. MIP-based heuristics for capacitated lotsizing problems. International Journal of Production Economics 85.

[22] Gu“ nterFandel ,CathrinStammen-Hegene. Simultaneous lot sizing and scheduling for multi-product multi-level production 140/AUZ II, 58084 Hagen, Germany.

[23] Knut Haase. The capacitated lot-sizing and scheduling problem with sequence-dependent setup costs and setup times. 